\title{
A Planck-scale limit on spacetime fuzziness and stochastic Lorentz invariance violation
}

\author{
Vlasios Vasileiou ${ }^{1 \star \dagger}$, Jonathan Granot ${ }^{2 \star}$, Tsvi Piran $^{3 \star}$ and Giovanni Amelino-Camelia ${ }^{4}$
}

\begin{abstract}
Wheeler's 'spacetime-foam'1 picture of quantum gravity (QG) suggests spacetime fuzziness (fluctuations leading to nondeterministic effects) at distances comparable to the Planck length, $L_{\mathrm{PI}} \approx 1.62 \times 10^{-33} \mathrm{~cm}$, the inverse (in natural units) of the Planck energy, $E_{\mathrm{PI}} \approx 1.22 \times 10^{19} \mathrm{GeV}$. The resulting nondeterministic motion of photons on the Planck scale is expected to produce energy-dependent stochastic fluctuations in their speed. Such a stochastic deviation from the well-measured speed of light at low photon energies, c, should be contrasted with the possibility of an energy-dependent systematic, deterministic deviation. Such a systematic deviation, on which observations by the Fermi satellite set Planck-scale limits for linear energy dependence ${ }^{2}$, is more easily searched for than stochastic deviations. Here, for the first time, we place Planckscale limits on the more generic spacetime-foam prediction of energy-dependent fuzziness in the speed of photons. Using high-energy observations from the Fermi Large Area Telescope (LAT) of gamma-ray burst GRB090510, we test a model in which photon speeds are distributed normally around $c$ with a standard deviation proportional to the photon energy. We constrain the model's characteristic energy scale beyond the Planck scale at $>2.8 E_{\mathrm{PI}}\left(>1.6 E_{\mathrm{PI}}\right)$, at $95 \%(99 \%)$ confidence. Our results set a benchmark constraint to be reckoned with by any QG model that features spacetime quantization.
\end{abstract}

Significant advances in exploring QG-motivated phenomenology $^{3}$ have been achieved in the past decade. In some cases, it was possible to establish bounds on QG effects at the Planck scale. Among the possible QG effects, it is expected ${ }^{4-6}$ that the postulated foamy/fuzzy structure of spacetime at short distances would induce a stochastic effect, where two massless particles of equal energy travel the exact same distance in different times. In this work, we examine a model of spacetime foam ${ }^{6}$, in which quantum fluctuations of spacetime near the Planck scale induce stochastic variations in the speed of light, $v(E)=c+\delta v(E)$, where $\delta v(E)$ is random and distributed normally around zero with a standard deviation $\sigma_{v}(E)=\left(1+n_{\mathrm{s}}\right) / 2\left(E / \xi_{s, n_{\mathrm{s}}} E_{\mathrm{Pl}}\right)^{n_{\mathrm{s}}} c$. In this model, we observationally expect a bunch of photons of equal energy $E$ emitted simultaneously from a distant astrophysical source to propagate with different speeds and arrive at different times, normally distributed around the light travel time $T$ for $v(E)=c$, with a standard deviation $\sigma_{T}(E)=T_{\mathrm{c}} \sigma_{v}(E) / c$, where $T_{\mathrm{c}} \sim T$ is given $\mathrm{by}^{7}$ :

$$
T_{\mathrm{c}}=c \frac{\sigma_{T}(E)}{\sigma_{v}(E)}=\frac{1}{H_{0}} \int_{0}^{z} \frac{\left(1+z^{\prime}\right)^{n_{\mathrm{s}}}}{\sqrt{\Omega_{\Lambda}+\Omega_{M}\left(1+z^{\prime}\right)^{3}}} \mathrm{~d} z^{\prime}
$$

with $H_{0}=100 h \mathrm{~km} \mathrm{~s}^{-1} \mathrm{Mpc}^{-1}$ being the Hubble constant and $\left[\Omega_{\Lambda}, \Omega_{M}, h\right]=[0.73,0.27,0.71]$ the cosmological parameters we used.

This stochastic speed-of-light variation is in conflict with Lorentz invariance, the basic symmetry of Einstein's theory of relativity. Such a conflict is usually referred to as Lorentz invariance violation (LIV). Here, we examine a simple manifestation of 'stochastic LIV', in which the light-cone of special relativity still exists; however it is fuzzy. The dimensionless parameter $\xi_{s, n_{\mathrm{s}}}$ determines the energy scale $\xi_{s, n_{s}} E_{\mathrm{Pl}}$ of stochastic LIV, and the model-dependent parameter $n_{s}$ determines the leading-order energy dependence of the effect. Stochastic LIV should be distinguished from deterministic LIV, for which LIV-induced dispersion is exactly the same for all photons of the same energy $E$ (that is, it is systematic) and is given by $\delta v(E)=-S_{n_{\mathrm{d}}}\left(1+n_{d}\right) / 2\left(E / \xi_{d, n_{\mathrm{d}}} E_{\mathrm{Pl}}\right)^{n_{d}} c$, with $S_{n_{\mathrm{d}}}= \pm 1$ a modeldependent parameter.

Systematic LIV represents a more appreciable departure from the structure of present theories, as in it the special-relativistic lightcone does not merely acquire some fuzziness but is actually replaced by a new structure. Moreover, whereas one should expect all spacetime-foam models to inevitably produce some amount of lightcone fuzziness of the type that induces stochastic LIV, systematic LIV requires spacetime foam with very particular properties.

It follows immediately from Wheeler's picture of spacetime foam $^{1}$ that for a particle of energy $E$ propagating in the foam, the travel time $T$ from source to detector should be uncertain following a law that could depend only on the distance travelled, the particle's energy and the Planck scale, with leading-order form of the type $\delta T \sim x^{n} E^{m} / E_{\mathrm{Pl}}^{1+m-n}$, where $m$ and $n$ are model-dependent powers and $1+m-n>0$. QG phenomenology is at present focused mostly on effects suppressed at the (first power of the) Plank scale, as stronger suppression leads to even weaker effects ${ }^{4}$. Therefore, we focus on cases with $n=m$. The particular case $n=m=0$ is a rather natural option, which, however, cannot be tested, as it requires timing with Planck-time $\left(t_{\mathrm{Pl}} \approx 5.39 \times 10^{-44} \mathrm{~s}\right)$ accuracy. As will become clear shortly, the picture we focus on here corresponds to the next natural choice of $n=m=1$, that is, $\delta T \sim x E / E_{\mathrm{Pl}}$.

Even though the model of spacetime-foam effects examined here is rather natural, its applicability to specific QG theories is in most cases difficult to establish, as obtaining rigorous physical predictions within the various complex QG mathematical formalisms is typically challenging. However, this model does apply to the important class of QG formalisms based on the so-called 'Lie-algebra noncommutative spacetimes ${ }^{3,9}$, which feature non-commutativity of coordinates of type $\left[x_{\alpha}, x_{\beta}\right]=i R_{\alpha \beta}^{\gamma} x_{\gamma} / E_{\mathrm{Pl}}$. This class of QG for-

\footnotetext{
${ }^{1}$ Laboratoire Univers et Particules de Montpellier, Université Montpellier 2, CNRS/IN2P3, Montpellier 34095 Cédex 05, France. ${ }^{2}$ Department of Natural Sciences, Open University of Israel, 1 University Road, POB 808, Ra'anana 4353701, Israel. ${ }^{3}$ Racah Institute for Physics, The Hebrew University of Jerusalem, Jerusalem 91904, Israel. ${ }^{4}$ Dipartimento di Fisica, Sapienza Università di Roma and INFN, Sezione di Roma 1, Piazzale Aldo Moro, 2,00185 Roma, Italy. †Present address: Zopa Inc.90 Fetter Lane, London EC4A 1EN, UK. *e-mail: vlasisva@gmail.com; granot@openu.ac.il; tsvi@phys.huji.ac.il
} 


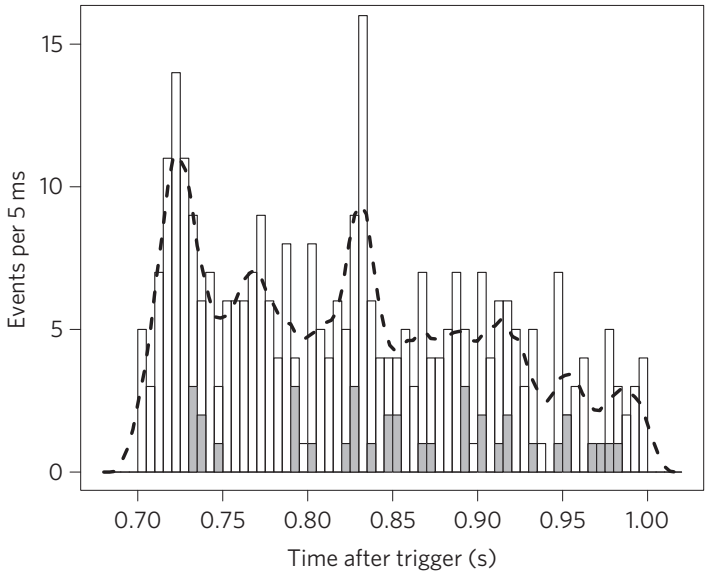

Figure 1 | Light curves of GRB090510. The white and shaded histograms show the light curves of GRB090510 below and above the threshold energy $E_{\text {th }}=300 \mathrm{MeV}$. The dashed curve shows the light curve template obtained using a kernel-density estimate of the emission below $E_{\text {th }}$.

malisms plays a key role in the only QG theory that has been solved so far-the dimensionally reduced $2+1 \mathrm{D}$ version of QG. Moreover, research on 3+1D QG has increasingly focused on Lie-algebra noncommutative spacetimes, for which the phenomenology examined here also applies.

To test the hypothesis of stochastic speed-of-light variations, we need bursts of photons of high energy and short duration observed from very distant sources. Gamma-ray bursts (GRBs) are ideal sources for this task ${ }^{10}$, thanks to their large distances (up to redshift ${ }^{11}$ $z \sim 9.4$ ), rapidly varying emission (minimum variability timescale down to $\mathrm{ms}$ ), and high-energy extent of their emission (up to tens of $\mathrm{GeV}$ ).

GRB090510 occurred at cosmological redshift ${ }^{12}$ $z=0.903 \pm 0.001$ and was one of the brightest GRBs ever detected ${ }^{13}$. It was an exceptional GRB with a short duration $(\sim 1 \mathrm{~s})$, a very high luminosity, photons of very high energy (up to $\sim 31 \mathrm{GeV}$ ), and a fine temporal structure with $\sim 10$ ms spikes in its light curve ${ }^{13,14}$. The best and most robust limits for both $n_{d}=1$ and $n_{d}=2$ deterministic LIV were obtained using observations of this burst ${ }^{2,15}$. Here, we use photon arrival time and energy data produced by the Fermi LAT (ref. 16) observations of GRB090510 to place a stringent constraint on $\xi_{s, 1}$. We consider only $n_{s}=1$, because for $n_{\mathrm{s}}=2$ the limits are several orders of magnitude away from the Planck scale, and thus not constraining QG models in a physically meaningful degree.

We adopt and modify for the case of stochastic effects a well-established maximum-likelihood analysis that was successfully used to search for deterministic LIV-induced energy dispersion in active galactic nuclei ${ }^{15,17,18}$ and GRBs (ref. 15; see Supplementary Information). We start the analysis by assuming that, without stochastic LIV, the detected GRB light curve would be identical across the whole observed Fermi-LAT energy range (from $\sim 10 \mathrm{MeV}$ to tens of $\mathrm{GeV}$ ). We then split the data into two energy ranges, separated by a threshold energy $E_{\text {th }}$. The photons below $E_{\text {th }}$ are used to estimate how the GRB emission's time profile would have been detected on average without LIV (the 'light curve template', $f(t)$ ), whereas the photons above $E_{\text {th }}$ are used for evaluating the likelihood function. The threshold energy $E_{\text {th }}$ is chosen to be low enough such that any dispersion effects below it are effectively negligible, yet high enough to allow adequate statistics for accurately estimating the light curve template. Based on simulations using synthetic data sets inspired by GRB090510, we chose $E_{\text {th }}=300 \mathrm{MeV}$; a choice that corresponds to increased sensitivity and minimal systematic biases.

We focus on the brightest, most variable time interval possessing the highest-energy photons of the GRB's prompt emission. To minimize systematic biases, we then analyse a sub-interval of it, during

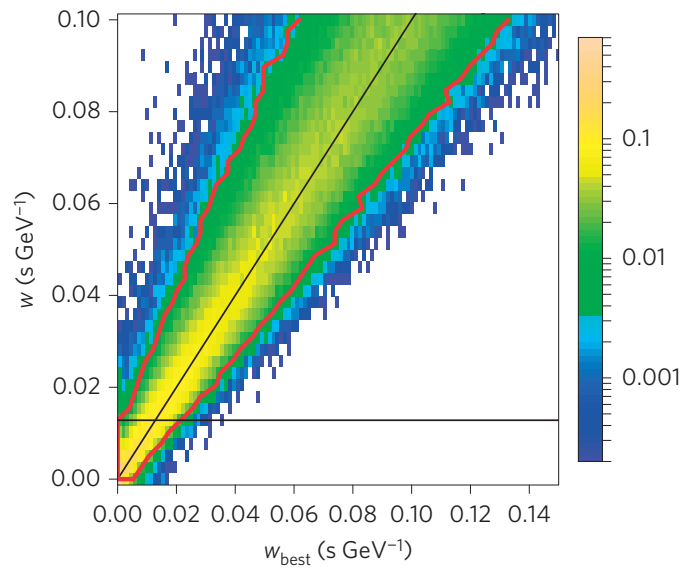

Figure $\mathbf{2}$ | Construction of the Feldman-Cousins confidence belt. Probability of obtaining a particular value of $w_{\text {best }}(x$-axis) versus the simulated value of $w$ ( $y$-axis). The pair of red curves shows the $95 \%$ Feldman-Cousins confidence belt. The horizontal solid black line denotes our $95 \%$ upper limit on $w$, given the value $w_{\text {best }}=0 \mathrm{~s} \mathrm{GeV}^{-1}$ obtained using the actual data. The diagonal thin solid black line denotes the line of equality between the simulated value $(w)$ and the best estimate for this value $\left(w_{\text {best }}\right)$.

which the GRB energy spectrum was measured to be relatively stable $^{13}$, namely $0.7-1.0 \mathrm{~s}$ post-trigger. Our chosen time interval and $E_{\text {th }}$ correspond to 316 photons below $E_{\text {th }}$ and 37 photons above $E_{\text {th }}$.

Guided by simulations, we estimate the light curve template using a 6-ms bandwidth kernel-density estimate of the detected emission below $E_{\text {th }}$. Figure 1 shows the estimated light curve template along with histograms of the arrival times of photons with energies below and above $E_{\text {th }}$. Using a maximum-likelihood analysis, we search for any dispersion in the high-energy part of the data (above $E_{\mathrm{th}}$ ) that is in excess of that below $E_{\text {th }}$. Assuming no source-intrinsic effects, we interpret any excess dispersion at high energies as arising from spacetime-foam effects, and use its presence or lack thereof to constrain these effects. For the examined case of $n_{\mathrm{s}}=1$, we quantify the magnitude of LIV-induced dispersion using the quantity $w(z)=\sigma_{T}(E) / E$. According to our maximum-likelihood analysis, the best estimate for $w$ is $w_{\text {best }}=0 \mathrm{~s} \mathrm{GeV}^{-1}$. This null result corresponds to the complete absence of any measurable excess dispersion in the high-energy part of the data.

To produce a confidence interval on $w$ we employ the FeldmanCousins (FC) approach ${ }^{19}$, as it provides confidence intervals of proper coverage and is also less sensitive to biases in the estimation of $w_{\text {best }}$. The implementation of the FC approach involved a set of simulations in which we first injected data sets similar to that of GRB090510 with various degrees of stochastic dispersion $(w)$, and then applied the maximum-likelihood analysis to measure this dispersion (that is, derive $w_{\text {best }}$ ). By examining the distribution of measured dispersion values $\left(w_{\text {best }}\right)$ for each particular value of the injected dispersion $(w)$, we found which values of true dispersion are possible given our measurement of $w_{\text {best }}=0 \mathrm{~s} \mathrm{GeV}^{-1}$, thereby producing a confidence interval on $w$. Figure 2 shows the results of these simulations along with the resulting FC confidence belt, which we used to place the constraints of $w_{\text {best }}<0.013(0.023) \mathrm{s} \mathrm{GeV}^{-1}$ at 95\% (99\%) confidence. These constraints on $w$ for the redshift of GRB090510, $z=0.903$, correspond to constraints of $\xi_{s, 1}>2.8$ (1.6) at $95 \%(99 \%)$ confidence, respectively. These are the first-ever constraints on spacetime-foam-induced stochastic variations on the speed of light that are beyond the physically important milestone of the Planck scale.

We extensively tested the validity and robustness of our results (see Supplementary Information). Specifically, we considered how the choice of time interval, the statistical uncertainty in the 
determination of the light curve template, the presence of spectral evolution, the imperfect energy reconstruction, the uncertainty of the cosmological parameters, and our choice of $E_{\mathrm{th}}$ affect the results. All the effects we examined had little influence on our results, shifting our upper limits by less than $10 \%$ (or $20 \%$ at worst, for a smaller time interval, $0.8-1.0$ s post-trigger), and often by much less, supporting the robustness of our results.

GRBs will probably remain the most constraining sources for direct searches of stochastic speed-of-light variations. Of course, one might alternatively seek indirect evidence of such variations: for example, based on the effect of these variations on the fuzziness of distance measurements operatively defined in terms of photon travel times, $L \equiv v T$. In this case, contributions to the uncertainty $\delta L \simeq c \delta T+T \delta v$ in $L$ would include in addition to the standard contribution from the Heisenberg uncertainty principle for a relativistic probe, $\delta T \geq \hbar / 2 E=\left(L_{\mathrm{Pl}} / 2 c\right) E_{\mathrm{Pl}} / E$ (where $\hbar$ is the reduced Planck constant), a novel contribution due to the stochastic QG effects:

$$
\delta L \simeq \frac{1}{2}\left(\frac{E}{E_{\mathrm{Pl}}}\right)^{-1} L_{\mathrm{Pl}}+\left(\frac{E}{\xi_{s, n} E_{\mathrm{Pl}}}\right)^{n_{\mathrm{s}}} L
$$

Equation (1) describes a fuzzy spacetime as there is no value of the energy of the probe for which the distance determination is classically sharp $\left(\delta L(E) \succsim \delta L_{\min }>0\right)$. Minimization with respect to $E$ implies (up to numerical factors of order unity), $\delta L \succsim\left(L_{\mathrm{Pl}}\right)^{n_{s} /\left(n_{s}+1\right)} L^{1 /\left(n_{s}+1\right)}$. Satisfactorily, this falls within the general expectations for the form of distance fuzziness $\delta L \succsim\left(L_{\mathrm{Pl}}\right)^{\alpha} L^{1-\alpha}$ discussed in previous works $\mathrm{s}^{4,5,20,21}$.

Equation (1) bridges two areas of investigation in quantum gravity: one on stochastic speed-of-light variations and one on a minimum-uncertainty principle for distance measurements. For purposes of exploiting this to perform indirect tests for stochastic speed-of-light variations, one should take notice that the best method to date for testing distance fuzziness relies on its implications on the formation of halo structures in the images of distant quasars ${ }^{5}$. However, the outcome of those studies depends rather crucially ${ }^{5}$ on the particular form of the fluctuations of the light-cone in the direction transverse to the one of propagation, in the same sense that our stochastic speed-of-light variations could be modelled purely in terms of fluctuations of the light-cone along the direction of propagation. Any attempt to test stochastic speedof-light variations on the basis of such quasar-image studies would only produce 'conditional limits', affected by assumptions about the fluctuations of the light-cone in the direction orthogonal to the propagation. A very exciting prospect for the future is to combine the type of study of stochastic speed-of-light variations proposed here and the studies of distance fuzziness developed in refs 5,20,21 to possibly obtain complementary views (or constraints) on lightcone fluctuations, both in the direction of motion and also in the transverse direction.

Remarkably, in spite of the stochastic nature of the examined dispersion, it was possible to obtain limits that are beyond the Planck scale, and comparable to those obtained for deterministic LIV (refs 2,15). These stringent limits should be taken into account when considering any QG theory that involves spacetime quantization. Using the methodology presented here, Fermi-LAT observations of GRBs brighter than GRB090510, and future high-sensitivity and higher-energy Cherenkov Telescope Array ${ }^{22,23}$ observations of short-duration GRBs would enable us to reduce even further the allowed windows for QG models.

Received 10 August 2014; accepted 2 February 2015; published online 16 March 2015

\section{References}

1. Wheeler, J. in Relativity, Groups and Topology (eds DeWitt, C. M. \& DeWitt, B. S.) 467-500 (Gordon and Breach, 1964).
2. Abdo, A. A. et al. A limit on the variation of the speed of light arising from quantum gravity effects. Nature 462, 331-334 (2009).

3. Amelino-Camelia, G. Quantum-spacetime phenomenology. Living Rev. Relativ. 16, 5 (2013).

4. Amelino-Camelia, G. Gravity-wave interferometers as quantum-gravity detectors. Nature 398, 216-218 (1999).

5. Christiansen, W., Ng, Y. J. \& van Dam, H. Probing spacetime foam with extragalactic sources. Phys. Rev. Lett. 96, 051301 (2006).

6. Amelino-Camelia, G. \& Smolin, L. Prospects for constraining quantum gravity dispersion with near term observations. Phys. Rev. D 80, 084017 (2009).

7. Jacob, U. \& Piran, T. Lorentz-violation-induced arrival delays of cosmological particles. J. Cosmol. Astropart. Phys. 01, 031 (2008).

8. Majid, S. \& Ruegg, H. Bicrossproduct structure of $\kappa$-Poincare group and non-commutative geometry. Phys. Lett. B 334, 348-354 (1994).

9. Madore, J., Schraml, S., Schupp, P. \& Wess, J. Gauge theory on noncommutative spaces. Eur. Phys. J. C16, 161-167 (2000).

10. Amelino-Camelia, G., Ellis, J., Mavromatos, N. E., Nanopoulos, D. V. \& Sarker, S. Tests of quantum gravity from observations of $\gamma$-ray bursts. Nature 393, 763-765 (1998)

11. Cucchiara, A. et al. A photometric redshift of $z \sim 9.4$ for GRB 090429B. Astrophys. J. 736, 7 (2011).

12. McBreen, S. et al. Optical and near-infrared follow-up observations of four Fermi/LAT GRBs: redshifts, afterglows, energetics, and host galaxies. Astron Astrophys. 516, A71 (2010).

13. Ackermann, M. et al. Fermi observations of GRB 090510: A short-hard gamma-ray burst with an additional, hard power-law component from $10 \mathrm{keV}$ to $\mathrm{GeV}$ energies. Astrophys. J. 716, 1178-1190 (2010).

14. Ackermann, M. et al. The first Fermi-LAT gamma-ray burst catalog Astrophys. J. Suppl. Ser. 209, 11 (2013).

15. Vasileiou, V. et al. Constraints on Lorentz invariance violation from Fermi-Large Area Telescope observations of gamma-ray bursts. Phys. Rev. D 87, 122001 (2013)

16. Atwood, W. B. et al. The large area telescope on the Fermi gamma-ray space telescope mission. Astrophys. J. 697, 1071-1102 (2009).

17. Martınez, M. \& Errando, M. A new approach to study energy-dependent arrival delays on photons from astrophysical sources. Astropart. Phys. 31, 226-232 (2009)

18. Abramowski, A. et al. Search for Lorentz Invariance breaking with a likelihood fit of the PKS 2155-304 flare data taken on MJD 53944. Astropart. Phys. 34, 738-747 (2011)

19. Feldman, G. J. \& Cousins, R. D. Unified approach to the classical statistical analysis of small signals. Phys. Rev. D 57, 3873-3889 (1998).

20. Lieu, R. \& Hillman, L. W. The phase coherence of light from extragalactic sources: Direct evidence against first-order Planck-scale fluctuations in time and space. Astrophys. J. 585, L77-L80 (2003).

21. Tamburini, F., Cuofano, C., Della Valle, M. \& Gilmozzi, R. No quantum gravity signature from the farthest quasars. Astron. Astrophys. 533, A71 (2011).

22. Actis, M. et al. Design concepts for the Cherenkov Telescope Array CTA: An advanced facility for ground-based high-energy gamma-ray astronomy. Exp. Astron. 32, 193-316 (2011).

23. Inoue, S. et al. Gamma-ray burst science in the era of the Cherenkov Telescope Array. Astropart. Phys. 43, 252-275 (2013).

\section{Acknowledgements}

The Fermi-LAT Collaboration acknowledges support for LAT development, operation and data analysis from NASA and DOE (United States), CEA/Irfu and IN2P3/CNRS (France), ASI and INFN (Italy), MEXT, KEK and JAXA (Japan), and the

K. A. Wallenberg Foundation, the Swedish Research Council and the National Space Board (Sweden). Science analysis support in the operations phase from INAF (Italy) and CNES (France) is also gratefully acknowledged. This research was supported by an ERC advanced grant (GRBs), by the I-CORE (grant No 1829/12), by the joint ISF-NSFC program (T.P.) and by the Templeton Foundation (G.A-C.).

\section{Author contributions}

All authors have contributed significantly to this work. V.V. and J.G. have focused mainly on the data analysis and deriving the limits, whereas T.P. and G.A-C. have focused mainly on the theory parts.

\section{Additional information}

Supplementary information is available in the online version of the paper. Reprints and permissions information is available online at www.nature.com/reprints. Correspondence and requests for materials should be addressed to V.V., J.G. or T.P.

\section{Competing financial interests}

The authors declare no competing financial interests. 